\title{
Presentación clínica de miastenia gravis en Atención Primaria: a propósito de un caso
}

M. A. Caballero Martín, R. Nieto Gómez, U. BarciK* Residente de $3^{\text {er }}$ año de Medicina de Familia y Comunitaria. C.S. Torrelodones. Madrid.

*Residente de $3^{\text {er }}$ año de Neurología. Servicio de Neuro-radiología. Clínica Ntra. Señora del Rosario. Madrid

\section{RESUMEN}

La Miastenia Gravis (MG) es una de las patolo gías neuromusculares más frecuentes. Su origen es autoinmune, y sus síntomas principales son la debi lidad y la fatigabilidad muscular, que afectan sobre todo a músculos faciales y proximales. El debút clí nico de la MG puede ser muy inespecífico y mime tizar cuadros muy diversos. El médico de familia debe tener presente la MG ante cualquier sintoma cuyo origen pueda deberse a una debilidad muscu lar. Su pronóstico depende de un rápido diagnósti co y tratamiento. Recordamos, a través de nuestro caso esta interesante enfermedad.

Palabras clave: Miastenia gravis. Presentación clínica. Debilidad muscular.

\section{Clinical presentation of myasthenia gravis in Primary Health Care: a case report}

\section{ABSTRACT}

Myasthenia Gravis (MG) is one of the most fre quent neuromuscular diseases. There is an autoin munological origin. The main symptoms are fatigue and muscle weakness which affects primarily mimic and proximal muscles. The inicial clinical presen tation can be very nonspecific and mimic various pathologies. However, a general practicioner should think about MG when a patient complains about muscle weakness. The prognosis depends on the correct diagnosis and treatment.

This case report wants to remind that interesting disease.

Key words: Myasthenia gravis. Clinical presen tacion. Muscle weakness.

\section{INTRODUCCIÓN}

La Miastenia Gravis (MG) es el trastorno adquirido crónico más frecuente de la unión neuromuscular, con una prevalencia aproximada en Europa Occidental de 8 a 15 casos por 100.000 habitantes $^{1,2}$.

Su presentación por edad es bifásica, con el máximo pico entre los 20 y 30 años y otro por encima de los 50. Es mas común en mujeres (3:2). Se trata posiblemente de la enfermedad de origen autoinmune mejor caracterizada.

Los síntomas principales son la debilidad y fatigabilidad de las fibras musculares esqueléticas que afecta sobre todo a grupos musculares proximales (cintura escapular y pelviana) y a los inervados por pares craneales, produciendo típicamente diplopia, claudicación mandibular y dificultad deglutoria. Esta clínica es característicamente fluctuante a lo largo del día, mejorando con el reposo y el sueño ${ }^{3}$.

El diagnóstico se basa en la exploración neurológica ante una clínica sugestiva, confirmándose mediante estudio inmunológico y electromiográfico.

Se trata de una patología poco frecuente y su método diagnóstico parece claro. Sin embargo queremos llamar la atención del médico de Atención Primaria, a través de nuestro caso. El mimetismo con otros procesos con los que la MG puede llegar a 
presentarse en los primeros estadios (los que el médico de primaria va a observar en primera instancia precisamente), hace, como veremos más adelante, que la realización de un diagnóstico correcto sea en ocasiones bastante difícil y nos lleve a pensar en múltiples y variados diagnósticos diferenciales.

\section{CASO CLÍNICO}

Se trata de una mujer de 48 años, fumadora de 30 cigarrillos al día, sin otros antecedentes patológicos de interés, ni alergias conocidas.

Refiere que el proceso comenzó con sensación de debilidad generalizada, astenia y síntomas subjetivos de desinterés y anhedonia. Estos síntomas aumentan con el transcurso del día, atribuyéndolo la propia paciente a un exceso de trabajo y estrés emocional. Durante semanas la debilidad progresa hasta el punto de hacerle sentarse tras subir las escaleras de un sola planta de su casa.

Dos meses después del inicio de la sintomatología, la paciente consulta por visión borrosa que aumenta con la luminosidad y el esfuerzo, mejorando con el reposo nocturno. Pocos días después de notar estas alteraciones visuales se añade claudicación mandibular con la masticación y dificultad para la elevación de brazos. No presenta síntomas de afectación respiratoria, digestiva ni urinaria. Tampoco refiere pérdida de peso reciente.

A la exploración física el estado general es bueno. Piel y faneras normales, no presenta bocio, la temperatura es de $36,5^{\circ} \mathrm{C}$ y la TA de $120 / 80$. Sin hallazgos en la auscultación cardiopulmonar y la palpación abdominal. Se descubre leve ptosis bilateral que aumenta con los tests de fatiga (elevación de la mirada a un punto fijo, parpadeo repetido), signo de párpado caído positivo y diplopia. El resto de pares craneales es normal. Presenta además disminución de fuerza en cintura escapular y pelviana simétrica que también se objetiva por fatiga a los pocos segundos de mantener brazos o piernas en elevación. Reflejos y sensibilidad son normales. Ante la sospecha de miopatía y para descartar posible patología maligna intracraneal expansiva, se deriva vía urgente al hospital de referencia.

Se realiza estudio complementario. Hemograma, VSG y coagulación son normales, así como el ECG. Presenta Ac. Anti-FML y Ac. Anti-FME positivos. El resto de estudio inmunológico (ANA, anti-LKM, anti-mitoc, anti-tiroideos, crioglobulinas, FR, IEF), hormonal (T4, THS), marcadores tumorales (CEA, AFP, B2HCG), bioquímica (B12, batería básica, orina), serologías (HIV, HCV, HBV, MAT, EBV, HSV, HZV, CMV, toxoplasma, micoplasma) es normal o negativo.

Las pruebas funcionales respiratorias revelan un patrón levemente obstructivo (FVC 81\%, FEV1
77\%, PIM 97, PEM 104). En la Rx de tórax se observa una masa en mediastino anterior de $7 \mathrm{~cm}$ que se confirma con TAC.

El estudio neurofisiológico es compatible con alteración de la unión neuromuscular. Se realiza una prueba de respuesta terapéutica con piridostigmina oral (MESTINOM) con el resultado de mejoría clínica muy significativa.

Con el diagnóstico de $\mathrm{MG}$ comienza tratamiento con anticolinesterásico, corticoide y la masa mediastínica se extirpa. El diagnóstico anatomopatológico es de timoma de predominio linfocítico con hiperplasia tímica asociada.

Dos años después del diagnóstico, la paciente continúa con corticoterapia a dosis descendentes y anticolinesterásicos a demanda estando asintomática la mayoría del tiempo.

\section{DISCUSIÓN}

En estudios epidemiológicos recientes se observa un aumento de la prevalencia de MG en nuestro medio, sobre todo en varones mayores de 50 años y con una mayor asociación a timomas ${ }^{4}$. Esto se debe a unos mejores medios diagnósticos, al envejecimiento poblacional ${ }^{1} \mathrm{y}$ al avance terapéutico que hace que se cronifiquen muchos de los casos al disminuir su mortalidad ${ }^{2}$.

En el $90 \%$ de los casos se detectan anticuerpos IgG anti receptor nicotínico de la acetilcolina en la membrana postsináptica de la unión neuromuscular ${ }^{5}$. Esto se traduce en dificultad de depolarización de dicha membrana a pesar de que la acetilcolina es liberada con normalidad. Si la afectación es sólo ocular los anticuerpos aparecen en el $50 \%$. Su negatividad no excluye el diagnóstico. En algunos casos estos anticuerpos tienen su origen en reacciones inmunológicas cruzadas con dominios proteicos que comparten el receptor de acetilcolina con proteínas de virus tan comunes como el herpes simplex ${ }^{6}$. Existe la posibilidad de inducción medicamentosa (D-penicilamina, procainamida, aminoglucósidos).

Histológicamente, el trastorno fundamental es una disminución en el número de receptores de acetilcolina y un aplanamiento de los plieges en la membrana postsináptica.

A pesar de que los detalles del origen de la síntesis de estos autoanticuerpos permanece sin esclarecer $^{7}$, existen evidencias del papel del timo en su formación. Más del 70\% de los pacientes presentan hiperplasias tímicas y hasta un 10-15\% timomas. Probablemente la reacción autoinmune comience en este órgano, pero las células T reactivas emigran en estadios muy precoces a otras localizaciones ${ }^{8}$. Cuando la enfermedad debuta en mayores de 50 años existe la particularidad inmunológica de pre- 
sencia de anticuerpos anti-músculo estriado en el $50 \%$ de los casos $^{9}$. Este autoanticuerpo es considerado marcador de existencia de timoma asociado ${ }^{10}$.

Escalas como la de Osserman y otras desarrolladas posteriormente nos ayudan a clasificar el grado de incapacidad de cada grupo muscular y su progresión ${ }^{11,12}$.

En un 10\% existe sólo afectación ocular. En raras ocasiones la debilidad afecta la musculatura respiratoria, siendo el pronóstico muy grave (crisis miasténicas).

El tratamiento consiste en la combinación de esteroides, anticolinesterásicos y en caso de gravedad, plasmaferesis e inmunosupresores. La timectomía está indicada en caso de timoma para evitar complicaciones de crecimiento local en el mediastino ${ }^{8}$, no como terapia curativa ya que según algunos estudios recientes no varía de forma significativa el curso de la enfermedad ${ }^{13}$.

La paciente que presentamos padeció síntomas muy característicos de miastenia gravis como diplopia (que se presenta en el $60 \%$ de los casos) 10 cual nos ayudó a una buena orientación diagnóstica. Sin embargo, los síntomas iniciales fueron inespecíficos y confusos.

La miastenia gravis puede simular gran cantidad de enfermedades neuromusculares (neuropatías, miopatías mitocondriales, síndrome de LambertEaton).

Se encuentran en la literatura casos de pseudoobstrucciones intestinales repetidas sin otros síntomas que son secundarias a parálisis de la pared intestinal, diagnosticados posteriormente de MG con hiperplasia tímica ${ }^{14}$. Otros casos han debutado con acalasia cuya patogenia parece ser la pérdida de las neuronas del plexo mientérico por mecanismo autoinmune asociado a timoma ${ }^{15}$. Incluso pacientes que sólo han referido disfonía crónica han resultado ser parálisis de cuerdas vocales (alguno precisando traqueotomía de urgencia) debidas a $\mathrm{MG}^{16,17}$.

En pacientes ancianos o afectos de alguna enfermedad neurodegenerativa, el diagnóstico diferencial de una debilidad en un grupo muscular concreto es aún más difícil, confundiéndose incluso con accidentes cerebro-vasculares ${ }^{18}$.

También encontramos casos diagnosticados durante años de psicopatologías, por comenzar su enfermedad con síntomas como cansancio generalizado, lentitud a la masticación y dificultad para la deglución que lleva a una negación de la ingesta ${ }^{19}$, debilidad para la elevación de los brazos (disminución del aseo y peinado diario), debilidad de cintura pelviana (evitación de la deambulación) y disminución de la mímica facial. De hecho hasta un $20 \%$ son confundidas en su debút con patologías psiquiátricas ${ }^{20}$, siendo el grupo de mujeres jóvenes el más perjudicado de este retraso diagnóstico. Además, la percepción subjetiva de debilidad muscular que tiene el paciente puede provocar ansiedad, depresión e incluso fobia social ${ }^{21}$ cuando la afectación de músculos faciales es grave.

Esta dificultad para orientar los sutiles primeros síntomas retrasa enormemente un diagnóstico y por tanto un tratamiento correcto, esencial para si no la curación, sí alcanzar una mejor calidad de vida y mejor pronóstico final ${ }^{22}$.

Así, es imprescindible, ante la aparición de cualquiera de los mencionados síntomas, realizar una historia clínica minuciosa, detallando momento de origen de los mismos, evolución, enfermedades o síntomas concomitantes. No hay que olvidar que MG también puede presentarse como síndrome paraneoplásico (más frecuentemente asociado a adenocarcinoma de pulmón), y que en ocasiones precede en años al diagnóstico del tumor ${ }^{23,24}$.

Hacer una exploración neurológica completa, comprobando que todos los reflejos y sensibilidad son normales, y aislando los grupos musculares afectos con tests de fatigabilidad muscular sencillos (repetición de tareas: abrir-cerrar ojos, abrir-cerrar boca, elevar brazos, etc.) es fundamental.

Entre los diagnósticos diferenciales que el médico de familia debe realizar se encuentra el hipertiroidismo, fácilmente excluible con pruebas de función tiroidea; el síndrome de Lambert-Eaton, en el cual objetivaremos una disminución de reflejos y/o alteraciones autonómicas; la neuroastenia y lesiones intracraneales ocupantes de espacio. En el caso de diplopia aislada pensaremos en posibles causas locales, miositis o infiltraciones orbitarias o lesiones de pares craneales oculomotores.

Teniendo presente la posibilidad de MG por parte del médico de familia y con una historia y exploración adecuadas, la confirmación de la sospecha es fácil con la búsqueda de anticuerpos antirreceptor de acetilcolina en suero y la electromiografía.

\section{AGRADECIMIENTOS}

A mi tutora, Fátima Bermejo, y a mi primera enfermera Charo, por darme ideas para trabajar.

CORRESPONDENCIA:

M. A. Caballero Martín

C. S. Torrelodones

Avda. de la Dehesa, s/n

28250 Torrelodones

Madrid

Tfno.: 918591474

Fax: 918593702

E-mail: marmariam@hotmail.com 


\section{Bibliografía}

1. Robertson NP, Deans J, Compston DA. Myasthenia gravis: a population based epidemiological study in Cambridgeshire, England. Neurol Neurosurg Psychiatry 1998; 65: 492-6.

2. Phillyps LH, Torner JC. Epidemiologic evidence for a changing natural history of myasthenia gravis. Neurology 1996; 47: 1233-8.

3. Drachman DB. Miastenia grave. En: Harrison's. Principios de Medicina Interna. $14^{\mathrm{a}} \mathrm{ed}$. Interamericana Mac Gray-Hill, 1998.

4. Vega-Garces WJ, Aguilera-Pacheco O, Núñez-Gil M, Luis-González S. Miastenia gravis en pacientes mayores de 50 años. Rev Neurol 1999; 28: 948-51.

5. Linstrom JM, Seybold ME, Lennon VD, Wittingham S, Drake D. Antibody to acetylcoline receptor in myasthenia gravis. Neurology 1976; 26: 1054-9.

6. Schimmbeck PL, Dyrberg T, Prachman DB, Olstone M. Molecular mimicry and miasthenia gravis. J Clin Invest 1989; 84: 1174-80.

7. Levinson AI, Wheatley LM. The thymus and the pathogenesis of myasthenia gravis. Clin Inmunology 1996; 78: 15.

8. Muller-Hermellin HK, Marx A. Pathological aspects of malignant and benign thymic disorders. Ann Med 1999; 31: 5-14.

9. Aarli JA. Late-onset myasthenia gravis: a changing scene. Arch Neurol 1999; 56: 25-7.

10. Aarli JA, Stefansson K, Marton LS, Wallmann RL. Patients with myasthenia gravis and thymoma have in their sera IgG autoantibodies against titin. Clin Exp Inmunol 1990; 82: 284-8.

11. Osserman KE, Genkins G. Studies in myasthenia gravis: review of a twenty year experience in over 1.200 patients. Mt Sinai J Med 1971; 38: 497-537.
12. D'Alessandro R, Casmiro M. Reliable disability scale for myasthenia gravis sensitive to clinical changes. Acta Neurol Scand 1995; 92: 77-82.

13. Werneck LC, Cunha FB, Scola RH. Myasthenia gravis: a retrospective study comparing thymectomy to conservative treatment. Acta Neurol Scand 2000; 101: 41-46.

14. Anderson NE, Hutchinson DO, Nicholson I, Aitcheson F, Nixon JM. Intestinal pseudo-obstruction, myasthenia gravis and thymoma. Neurology 1996; 47: 985-7.

15. Henry J, Kaminsk MD. Achalasia and myasthenia gravis in a patient with thymoma. Neurology 1999; 52: 425-6.

16. Victor Osei-lah MB, O'reilly BJ. Bilateral abductor vocal fold paralysis due to myasthenia gravis. J Laryng Otol 1999; 113: 678-9.

17. Putman MT, Wise RA. Myasthenia gravis and uper airway obstruction. Chest 1996; 109: 400-4

18. Kleiner-Fisman G, Stephen Kott H. Myasthenia gravis mimicking stroke in elderly patients. Mayo Clin Proc 1998; 73: 1077-8.

19. Hanson JA, Lueck CJ, Thomas DJ. Myasthenia gravis presenting with stridor. Thorax 1996; 51: 108-9.

20. Rohr W. Myasthenia gravis in the frontier of psychiatric diagnosis. Psychiatr Prax 1992; 19: 157-63.

21. Rohr W. Situational anxiety in myasthenia gravis. Psychother Psychosom Med Psychol 1993; 43: 93-9.

22. Cosi V, Romani A, Lombardi M, Paiola E . Prognosis of myasthenia gravis, a retrospective study of 380 patients. J Neurol 1997; 244: 548-55.

23. Fujita J, Yamadori I, Yamaji Y. Myasthenia Gravis associated with small cell carcinoma of the lung. Chest 1994; 105: 624-5.

24. Ramos-Leo YL, Reyes CV. Myasthenic syndrome (EatonLambert syndrome) associated with pulmonary adenocarcinoma. J Surg Oncol 1987; 34: 239-42. 